site sur cet emplacement, au voisinage immédiat du nerf optique et du cerveau, a une importance particulière. Au reste, Nybelin a trouvé l'Aphanomyces dans le cerveau et les ganglions viscéraux de Crustacés dont la carapace était intacte. Quant à Scruxora, il indique les lamelles branchiales comme la voie d'accès du Champignon.

Il faut parfois de longues investigations pour déceler la présence de cet Oomycète bien nommé puisqu'en grec àparris veut dire : caché, secret. On doit le rechercher sur des préparations fraiches car la fixation l'altère à ce point qu'il devient fréquemment méconnaissable'.

Les caractères généraux de l'A. astaci concordent avec ceux donnés pour le genre par Von Minden et Lindau. Les hyphes qui s'entrecroisent dans la couche chitinigène sont très fins $(3,4: 5,6 \mu$ à l'état frais), ce qui les rend difficiles à apercevoir ; leur protoplasma est incolore et ils ont des extrémités arrondies. Mais ils sont plus ramifiés que chez le type, de là résulte, pour partie, l'aspect réticulé sous lequel se présente le thalle. Dans l'intérieur, on n'observe pas toujours de fines granulations, mais assez souvent des inclusions sphériques remarquablement grosses. Par fixation et coloration à l'hémalun on fait apparaitre force noyaux et vacuoles, mais leurs dimensions minimes les rendent peu apparentes sur les photographies (I).

(A suivre).

\title{
REMARQUES SUR LE SAUMON DE L'ADOUR
}

\section{Par M. Penne CHIMITS}

Garde général des Eaux et Porets.

(Suite) (2)

$$
\text { II. - Le SaUmon }
$$

A. - Hypothèse de la remontée dans la rivière d'origine.

La première question qui se pose est celle de savoir si le Saumon, qui a été smolt dans la Nive ou dans les Gaves, revient toujours dans la rivière dont il est originaire.

Mrnzirs est parvenu à démontrer que, pour les rivièrẹs écossaises, les

(1) Voir figure dans le prochain numéro.

(2) Voir Bulletin : - $N^{\circ} 99$, Septembre 1936, p. 57 . 
smolts marqués ont été repris comme Saumons toujours dans leur rivière de naissance, et en conclut à l'existence de races différentes de Saumons, sans toutefois avoir pu les distinguer par des caractères morphologiques.

Bien que, a priori, cette existence de races soit plus difficile à admettre dans le cas de l'Adour et de son affluent la Nive, les Saumons des deux rivières entrant forcément par la même embouchure pour se séparer à Bayonne, les résultats de la lecture des écailles semblent en faveur de cette théorie de la remontée du Saumon dans sa rivière d'origine, soutenue par M. le Commandant RocQ. En effet, dans le cas de la Nive, nous trouvons que 5 Saumons ont été smolts de $\mathrm{I}$ an et 6 de 2 ans sans trouver de smolts de 3 ans, et dans le cas des Gaves, 5 Saumons, smolts de 2 ans et 4 de 3 ans, sans trouver de smolts de 1 an, ce qui correspond bien aux proportions de tacons des divers âges dans les lots respectifs de tacons des Gaves et de la Nive. Mris cette question ne peut être tranchée que par des marquages de smolts que M. l'Inspecteur Principal LAfrieu compte faire cet hiver.

\section{B. - Mensurations morphologiques. - Sexe.}

Il n'a pu être fait de mensurations morphologiques sur le Saumon, étant donné qu'il ne parvenait au Laboratoire que les écailles, l'indication de la longueur totale et, dans le cas des Saumons de la Nive, le sexe du poisson.

Sur les 12 Saumons capturés dans la Nive, il est curieux de constater que ro sont des femelles, soit plus de $80 \%$ (alors que chez les tacons de 2 ans, $80 \%$ est la proportion des mâles).

Il importera de vérifier s'il s'agit là d'une proportion normale dans la Nive, ou s'il s'agit simplement d'un effet du hasard sur un petit nombre de captures.

$$
\text { C. - Facteur condition. }
$$

Le facteur condition $\mathrm{K}={ }^{100} \frac{t}{\mathrm{~L}}$ a été trouvé de 0,895 pour les Saumons de la Nive et de o,935 pour ceux des Gaves. Ces chiffres sont inférieurs à ceux donnés par Menzies pour les deux raisons suivantes :

$I^{\circ}$ La longueur mesurée sur le Saumon de l'Adour est la longueur totale, sur le Saumon écossais la longueur jusqu'à l'extrémité du plus court rayon de la caudale ;

$2^{\circ}$ Le poids des Saumons de l'Adour, ayant séjourné un temps variable en rivière sans s'alimenter, est inférieur, toutes choses égales d'ailleurs, au poids des Saumons écossais pris à leur montée.

On ne peut donc comparer ces chiffres avec ceux de Menzizs. 


\section{D. - Croissance thalassique.}

Voici le tableau de comparaison des Saumons de formule, p. 2 et p. 3 (I).

\begin{tabular}{|c|c|c|c|c|c|c|c|c|}
\hline \multirow{2}{*}{ 幽 } & \multicolumn{2}{|c|}{ NIVE } & \multicolumn{2}{|c|}{ GAVES } & \multicolumn{2}{|c|}{ BRETAGNE } & \multicolumn{2}{|c|}{ Ecosse } \\
\hline & Congueur & Poids & Longueur & Poids & Longueur & Poids & Longueur. & Poids \\
\hline p. 2 & 85 & 4,5 à 5,3 & 82 & $4,5 \& 5,35$ & $\begin{array}{c}77,5 \\
70 \text { a } 85)\end{array}$ & $5-7$ & 72 & 3,9 à 4,3 \\
\hline p.3 & 101 & 9,6 & 96 & 8,3 & $\begin{array}{c}95 \\
(90 \text { à } 100\end{array}$ & $8-12$ & 93 & 8,5 à 9 \\
\hline
\end{tabular}

Pour les raisons qui viennent d'être exposées au paragraphe précédent, on ne peut comparer, ni les poids des poissons des diverses provenances, ni la longueur des poissons d'Ecosse avec celles des trois autres catégories de Saumon.

Cependant, ce tableau montre que, comme pour le tacon, le Saumon de la Nive croft plus vite que celui des Gaves, qui croft plus vite que celui de Bretagne.

Ci-contre le graphique de croissance thalassique des Saumons qui ont été smolts 2 ans (p. 2) (Fig. r3). Le Saumon de la Nive, bien qu'ayant quitté la rivière à une taille légèrement plus petite croit plus rapidement au cours de la première année que le Saumon des Gaves et maintient par la suite son avance.

Si nous examinons maintenant le graphique (Fig. I4) de la croissance thalassique des Saumons r.3 et 3.3 nous voyons que le Saumon qui a émigré comme smolt dé $\mathrm{I}$ an $\mathrm{a}$ I $\mathrm{cm}$. 5 de longueur, rejoint presque en taille, au bout de la première année de croissance, le Saumon qui a émigré comme'smolt de 3 ans, ayant alors 21 cm. 5 de longueur. Par la suite, entre la deuxième et la troisième année, il le dépasse et revient en rivière avec une taille supérieure de $6 \mathrm{~cm}$. 5 . Par conséquent, le smolt de $\mathrm{I}$ an qui arrive dans le milieu marin, non seulement n'est pas dans une condition inférieure à celle des smolts de 2 et 3 ans, mais eqncore crolt plus vite et finit par les dépasser.

\section{E. - Age du sţolt et quantité de Saumons dans une rivière.}

De ces divers faits résulte un double bénéfice pour la rivière à Saumons qui, comme la Nive, a une certaine proportion de smolts de $I$ an.

D'abord ces smolts de. I an reviennent comme Saumons de plus grande taille.

(I) La farmule p. 2 indique un poiseon ayant passè plusieurs annés in rivière et 2 ans en riviere et. 2 ans en mer; la formule p. 3 un poisson ayant passé plusieurs années en riviére et 3 ans en mer. Voir page 59 . 


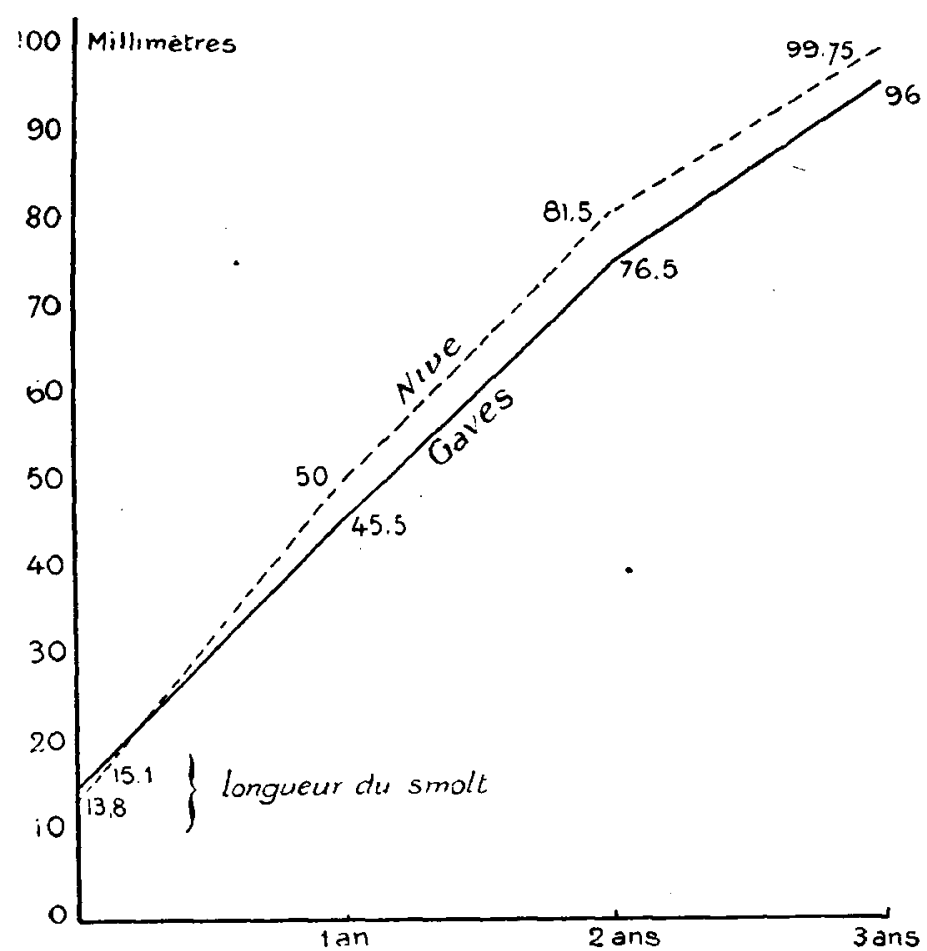

Fig. 13. - Croissance comparée dés Saumons de la Nive et des Gaves, qui ont été smolts à 2 ans.

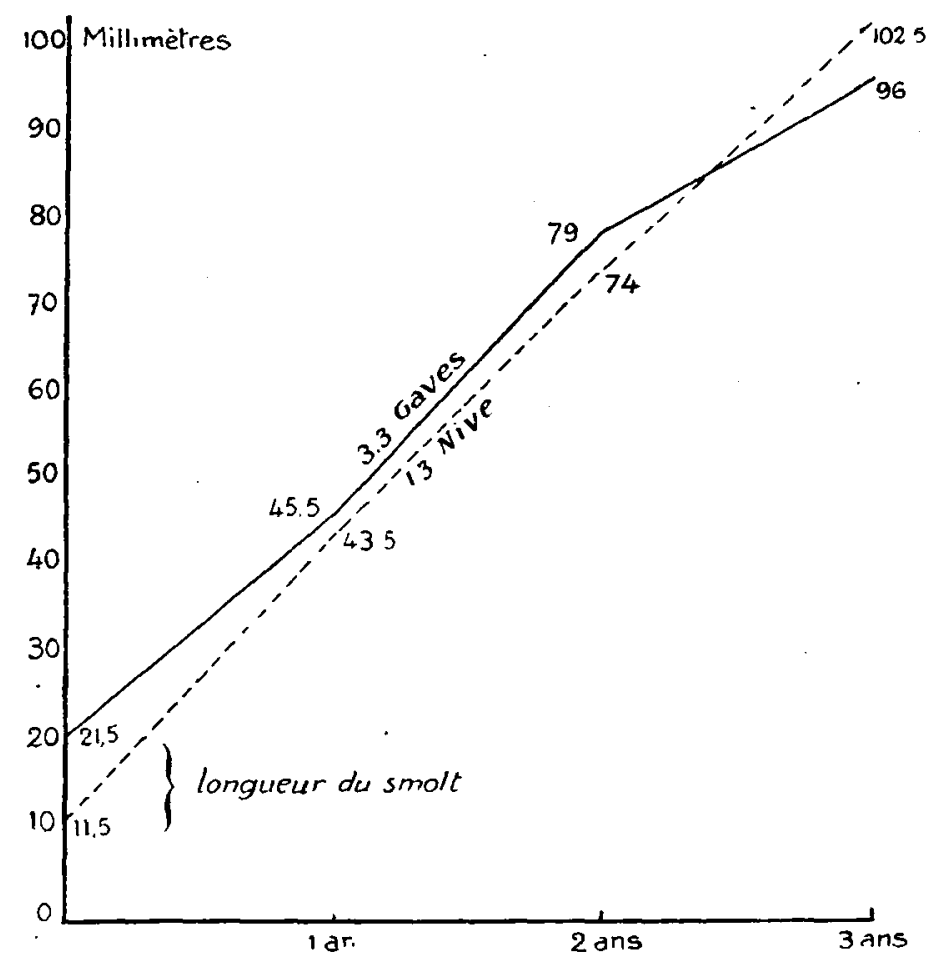

Fıg. 14. - Crois iance comparée des Saumons de la Nive et des Gaves, qui ont élí smolis 3 ans. 
Ensuite et surtouit, la capacité biogénique en tacons d'une rivière à smolts de $I$ an est théoriquement, toutes choses égales d'ailleurs, double de celle d'une rivière à smolts de 2 ans, triple de celle d'une rivière à smolts de 3 ans, puisque cette rivière, au lieu de nourrir à côté des tacons de l'année un contingent de tacons de l'année précédente et un autre de l'année avant, - réserve toute sa nourriture aux tacons qu'elle produit chaque année et qui émigrent tous chaque année. Par conséquent, si l'hypothèse du retour du Saumon à sa rivière natale est définitivement vérifiée dans le cas de l'Adour et de ses affluents, la Nive, toutes choses égales d'ailleurs, devrait recevoir plus de Saumons que les Gaves.

En d'autres termès, si nous revenons à l'exemple donné plus haut des Saumons de formule 1.3 de la Nive et 3.3 de l'Adour, nous voyons que :

La Nive a donné $\mathrm{I}$ an de nourriture de tacon pour obtenir un Saumon de $102 \mathrm{~cm} .5$.

L'Adour (ou mieux le Gave) a donné 3 ans de nourriture de tacon pour obtenir un Saumon de 96 centimètres.

Par conséquent, à capacité biogénique égale, et toutes choses égales d'ailleurs, la Nive devrait avoir trois fois plus de Saumons que les Gaves.

Il est superflu d'écrire que cette manière mathématique de présenter les choses est loin d'être l'image absolue de la réalité.

D'abord, il n'y a pas de rivières ayant uniquement des smolts de $\mathrm{I}$ an ou des smolts de 2 ans ou des smolts de 3 ans. La Nive a des smolts de I et 2 ans, l'Adour a des smolts de 2 et 3 ans.

Puis, le tacon n'est pas le seul poisson de ces rivières, le surplus de nourriture laissé par l'absence de tacons de 2 et 3 ans peut aussi bien être absorbé par la Truite ou tout autre poisson.

Enfin, la quantité d'aliments nécessaire à un tacon émigrant au bout de sa première année n'est certainement pas la même que celle nécessaire annuellement $\mathrm{a}$ un tacon de 2 ou 3 ans.

Aussi, n'y a-t-il là qu'une indication grossière, mais dont il faut cependant tenir compte, et qui augmente certainement pour la Nive la capacité biogénique en tacons, et par conséquent en Saumons, si l'hypothèse de la remontée à la rivière d'origine est vérifiée.

\section{F. - Ralentissement de croissance.}

Menzies dans son rapport au Congrès de Gdynia déclare que " un ralentissement dans le taux de croissance est souvent montré dans la croissance d'été et arrive fin Juin et aux premiers dix jours de Juillet, bien que certaines ańnées elle puisse avoir lieu plus tard".

Ceci est également vrai pour le Saumon de l'Adour.

Ce ralentissement de croissance se traduit par la formation d'une zone de stries semblable à une zone hivernale, si semblable qu'il est souvent difficile de les distinguer, ce qui n'est pas pour faciliter l'interprétation de l'écaille. 
On peut les distinguer ainsi :

$I^{\circ}$ Le plus souvent, la zone d'arrêt estivale est moins prononcée que la zone d'arrêt hivernale ;

$2^{\circ}$ La zone d'arrêt estivale ne porte pas de marque de ponte ;

$3^{\circ}$ S'il y a encore doute, la scalimétrie montre laquelle des deux zones donne un chiffre raisonnable pour la taille du saumon à l'année déterminée par la zone hivernale.

$$
\text { G. - Marques de ponte. }
$$

Il n'a pas été trouvé de marque de ponte dans le lot de Saumons des Gaves. Au contraire, sur 12 Saumons de la Nive, 3 femelles avaient déjà pondu une fois ( $1.2 G_{1} ;$ I. I $G_{\text {I }}$ et ${ }_{2 .}{ }_{2} G_{1}$ ) et une femelle avait déjà pondu deux fois (2. I G I G I).

Les marques de ponte sont en général faiblement marquées mais nettes, ce qui vient à l'appui de la théorie de $M$. le Commandant Roce et de l'article de M. Boursset.

" La bande de ponte est assez peu marquée, bien que nette.... ce qui " indique sans doute que, n'ayant qu'un faible parcours à effectuer pour " arriver à ses frayères, le Saumon de la Nive ne subit alors qu'une " déchéance organique peu avancée "( I).

Cependant, chez un individu de formule $I . x+G$, la marque de ponte est particulièrement nette. On peut peut-être l'expliquer ainsi : le poisson étant entré en rivière l'été $(\mathbf{I}+)$ est resté pour pondre jusqu'à l'hiver de l'année suivante.

Cette marque de ponte se traduit :

I Par une "éraillure ", c'est-à-dire l'apparition d'une bande de la couche inférieure fibreuse de l'écaille, la couche supérieure d'hyalodentine ayant disparue ;

$2^{\circ}$ Par l'interruption brutale des stries d'hyalodentine de la partie interne de l'écaille, au niveau de l'éraillure ;

$3^{\circ}$ Par la formation de stries externes et ultérieures, non concentriques avec les stries internes, l'aspect général pouvant se comparer à des couches géologiques en discordance ;

Ces trois caractères sont assez nets sur la première photographie ci-après (Fig. 15).

$4^{\circ}$ Par la formation d'une "poche de pọnte " bien marquée sur la seconde photographie (Fig. 16).

Cette " poche de ponte " confirme la théorie de Menzies sur la cicatrisation de la marque de ponte (2).

(1) Bulletin de la Société de la Nive, - Octobre rg27.

(2) Loco citato : chapitre I. 
Voici, semble-t-il, comment on peut l'expliquer :

Lors de la ponte, une éraillure profonde, en forme de poche, s'est formée sur l'écaille, coupant brusquement les stries normales:

Au retour du poisson à la mer, la cicatrisation s'est faite par la formation de nouvelles stries plus serrées d'hyalodentine en discordance complète

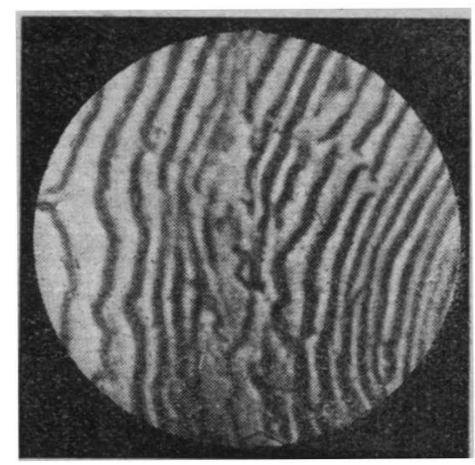

Frg. 15. - Ecaille de Saumon de la Nive. Marque de ponte. $\mathbf{G}=110$.

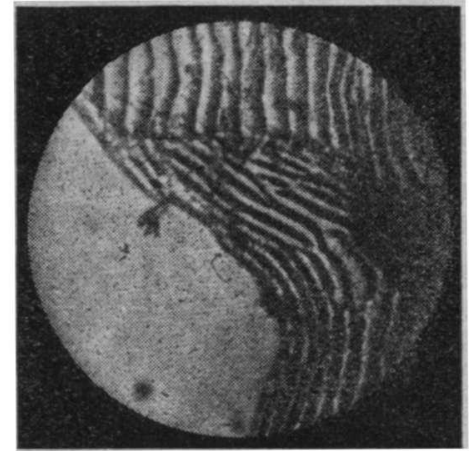

Frg. 16. - Saumon de la Nive. Poche de ponte. $\mathbf{G}=110$.

avec les stries normales et épousant la forme de la poche pour consolider le mieux possible la fracture.

Dans ce cas, la cicatrisation est parfaite, les nouvelles stries d'hyalodentine rejoignant les anciennes.

Cette formation de poche cicatrisée par des stries discordantes par rapport aux stries anciennes est un indice particulièrement net de la ponte.

\section{GoncLusion}

Telles sont les observations faites au cours du stage, du 2 Septembre au 8 Octobre rg35.

Si la différenciation morphologique, tout comme dans le cas des Saumons d'Ecosse, n'a pas jusqu'ici donné de résultats (sauf pour la différenciation possible de 2 races de tacons dans les Gaves), les faits consignés dans ce rapport favorisent l'hypothèse de races physiologiques différentes (par les phénomènes de sexualité, de croissance, d'âge du stade smolt, etc.) dans le cas des Gaves et de la Nive, se distinguant elles-mêmes des races d'Ecosse et de Bretagne. Il manque encore, à ces faits et à ces mensurations; l'appui de la loi des grands nombres.. Mais cette existence de races est subordonnée à l'hypothèse de la remontée du Saumon à la rivière originelle, qu'il reste encore à prouver de façon irréfutable.

Si on ajoute à cela la question de capacité biogénique, on voit qu'il y a fort à faire, pour connattre de façon certaine la biologie du Saumon de l'Adour. 